\title{
Avaliaçáo da Consciência Querológica de Crianças Surdas PorTuguesas: O IACQ-LGP ${ }^{1}$ \\ Assessment of Cherologic Awareness in Portuguese Deaf Children: the $I A C Q-L G P$
}

\author{
Marta PRATAS ${ }^{2}$ \\ Isabel Sofia Calvário CORREIA ${ }^{3}$ \\ Sofia SANTOS 4
}

\begin{abstract}
RESUMO: A avaliação da língua gestual Portuguesa (LGP) exige maior atenção à consciência querológica, dada a sua relevância na aprendizagem da língua. No âmbito nacional, constata-se a inexistência de instrumentos avaliativos da consciência querológica, pelo que o objetivo deste artigo é apresentar o Instrumento de Avaliaçâo da Consciência Querológica (IACQ-LGP) e a análise das suas propriedades métricas. O instrumento (constituído por 5 subtestes) foi aplicado a 23 participantes entre os 5 e 12 anos

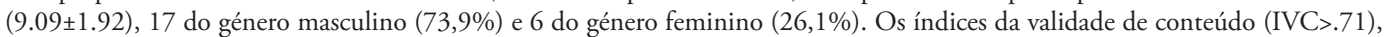
acordo universal (>.94) e média (>.94) apontaram a relevância dos itens. A proporçáo de acordo (.82 e 1) indiciou o forte consenso entre peritos, bem como os valores de Kappa de Cohen ( $\mathrm{k}>.84)$. A fiabilidade foi confirmada pela consistência interna $(.78>\alpha<.82)$ e correlaçóes moderadas a fortes ao nível da estabilidade temporal, pela técnica teste-reteste $(.37>\mathrm{r}<.73)$, exceção ao que avalia a discriminação de pares mínimos. A validade de constructo, analisada pelas intercorrelaçôes entre domínios apontou correlações fracas a moderadas $(.12 \geq \mathrm{r} . \leq 84)$, e a análise fatorial exploratória a multidimensionalidade do constructo. O IACQ-LGP parece ser um instrumento fiável e válido para avaliar a consciência querológica. Conclusóes e recomendaçóes serão apresentadas.
\end{abstract}

PALAVRAS-CHAVE: Avaliação. Surdo. Língua Gestual Portuguesa. Propriedades Psicométricas. Consciência Querológica. Educação Especial.

ABSTRACT: The assessment of Portuguese Sign Language (PSL) demands an increase interest to cherological awareness, due to its relevance for PSL learning. At the national level, there is a lack of instruments for evaluating kerological awareness, so the purpose of this article is to present the Kerological Awareness Assessment Instrument (Instrumento de Avaliação da Consciência Querológica - IACQ-LGP) and the analysis of its metric properties. The instrument (consisting of 5 subtests) was applied to 23 participants between 5 and 12 years old $(9.09 \pm 1.92), 17$ males $(73.9 \%)$ and 6 females $(26.1 \%)$. The content validity indexes (IVC> .71), universal agreement $(>.94)$ and average $(>.94)$ indicated the relevance of the items. The proportion of agreement (.82 and 1 ) indicated the strong consensus among experts, as well as Cohen's Kappa values ( $\mathrm{k}>.84)$. The reliability was confirmed by the internal consistency $(.78>\alpha<.82)$ and moderate to strong correlations in terms of temporal stability, by the test-retest technique $(.37>\mathrm{r}<.73)$, except for the one that evaluates the discrimination of pairs minimums. Construct validity, analyzed by intercorrelations between domains, showed weak to moderate correlations $(.12 \geq \mathrm{r} . \leq 84)$, and exploratory factor analysis showed the multidimensionality of the construct. The IACQ-LGP appears to be a reliable and valid instrument for assessing kerological awareness. Conclusions and recommendations will be presented.

KEYWORDS: Assessment. Deaf. Portuguese Sign Language. Psychometric Properties. Cherologic Awareness. Special Education.

\footnotetext{
${ }^{1}$ https://doi.org/10.1590/1980-54702021v27e0033

${ }^{2}$ Professora de Língua Gestual Portuguesa. Faculdade de Motricidade Humana. Universidade de Lisboa. Lisboa/Portugal. E-mail: marta.pratas@hotmail.com. ORCID: https://orcid.org/0000-0002-8570-2698

${ }^{3}$ Escola Superior de Educaçăo. Instituto Politécnico de Coimbra. Coimbra/Portugal. E-mail: isofia.cc@gmail.com. ORCID: https://orcid.org/0000-0002-1798-2165

${ }^{4}$ Unidade de Investigação e Desenvolvimento em Educação e Formação (UIDEF). Instituto da Educação. Faculdade de Motricidade Humana. Universidade de Lisboa. Lisboa/Portugal. E-mail: sofiasantos@fmh.ulisboa.pt. ORCID https://orcid.org/00000002-6654-564X
} 


\section{INTRODUÇÁo}

A avaliação do desenvolvimento linguístico passa, sobretudo, por averiguar (se, e) quais os aspetos linguísticos alterados, identificando o perfil desenvolvimental, tipo e monitorização de intervenção (Lima, 2011). Para o domínio da língua gestual, a criança deve dar atenção ao significado e não só às formas da mão, de forma precoce (Basso, 2006), reconhecendo que os gestos são formados por unidades menores, suscetíveis de serem isoladas e manipuladas, os queremas. A essa habilidade na Língua Gestual Portuguesa (LGP), equiparada à consciência fonológica da língua oral, atribui-se o nome de consciência querológica (Correia, 2009), definida como capacidade de refletir, analisar e manipular os segmentos mínimos que constituem um gesto, implicando a sua significação e parâmetros gestuais (Karnopp, 1977). O seu desenvolvimento demonstra-se em uma progressão de grandes unidades (palavras/gestos) para a consciência de pequenas unidades, ou queremas (Carrol et al., 2003). No programa curricular da LGP (Carmo et al., 2007), o ensino da consciência querológica inicia-se na educação pré-escolar, e para uma intervenção mais consistente e objetiva, exige-se a sua monitorização (Haug, 2005). No entanto, percebe-se que não há qualquer instrumento que avalie esta consciência no âmbito nacional e que, dada a sua importância linguística, o ensino da LGP poderá ficar afetado.

$\mathrm{Na}$ avaliação da Língua Gestual, o primeiro teste padronizado mundialmente foi o Assessing British Sign Language: Receptive skills test (RST - Herman et al., 1999). Os autores avaliaram 28 crianças surdas e 13 crianças ouvintes entre os 3 e os 11 anos, 21 do género masculino e 20 do género feminino, com o teste British Sign Language (BSL), medindo a compreensão da gramática da língua gestual inglesa. Da análise inicial, oito itens foram eliminados, mantendo-se 36 com valores de correlação a variar entre .20 e .80 (p<.05), apesar de três terem sido eliminados dada a pouca fiabilidade. A correlação entre respondentes (pais e professores) foi elevada (.99, p<.001), com valores de consistência interna (.90) e estabilidade temporal (.91) excelentes (Herman et al., 1999). Os autores reportaram a existência de diferenças significativas entre crianças entre os 3 e 6 anos com os grupos restantes (6-9 e 9-11 anos), reparando, no entanto, na inexistência de diferenças nos dois grupos mais velhos, eventualmente explicados pelo desenvolvimento gramatical estar adquirido por volta dos 8 anos. $\mathrm{O}$ género não aponta diferenças entre participantes $(\mathrm{p}=.29)$, bem como a comparação entre crianças surdas e ouvintes (.52). Na fase de normalização, a tendência manteve-se (Herman et al., 1999).

No mesmo ano e nos Estados Unidos da América (EUA), Maller et al. (1999) construíram o American Sign Language Proficiency Assessment (ASL-PA), cuja seleção de itens se baseou na revisão da literatura e no acordo de peritos (98\%). A avaliação envolveu 80 crianças, entre os 6 e 12 anos, 42 do género feminino, e os autores analisaram o nível de dificuldade dos itens, ao mesmo tempo que estabeleceram os valores de corte para a classificação da proficiência (baixa, moderada e alta). A fiabilidade foi analisada recorrendo aos coeficientes de KuderRichardson20, com valores entre .80 e .90 , e de consistência interna entre .75 e .85 . O teste diferenciou nativos e não-nativos de língua gestual $(\mathrm{p}<.05)$, apesar da inexistência de diferenças no que toca à idade e ao género (Maller et al., 1999).

Ao nível da avaliação da consciência querológica, o instrumento de avaliação da consciência fonológica e do parâmetro da mão para crianças surdas brasileiras (Cruz \& Lamprecht, 2008) foi aplicado a 15 crianças surdas entre os 6 e 11 anos com início da aquisição da língua entre os 0 meses e os 4 anos. O teste é constituído por duas partes: 1) proficiência lexical da 
configuração da mão (CM) - a criança deverá imitar o gesto que lhe sairá em um conjunto de 120 apresentadas, e os itens são cotados entre: denominação esperada, não esperada, esperada modificada com algum parâmetro modificado, e não denomina; 2) mais cinco itens com 30 tarefas (comparação da CM de um gesto com a CM dos restantes, evocação de gestos a partir de uma imagem), cotados com 0 (escolha errada da imagem) e 1 (escolha acertada da imagem). As propriedades psicométricas do instrumento não são apontadas, descrevendo-se apenas o nível dos acertos das respostas, concluindo que o período de exposição linguística influencia o desempenho.

Em 2010, na Holanda, Hermans, Knoors e Verhoeven propuseram o Assessment of Sign Language Development to Deaf Children para avaliar a proficiência da língua gestual de 330 crianças surdas, entre os 4 e 12 anos, em educaçáo bilingue, por meio de nove testes computadorizados, avaliando diferentes questóes linguísticas: querologia, vocabulário, morfossintaxe e narração. No âmbito da querologia, avaliou-se: a recetiva (qr) com 36 itens, e com os avaliados instruídos a responder se os gestos tinham (ou não) o mesmo significado depois de apresentados pares mínimos; e a expressiva (qe) com a apresentação de um gesto que a criança devia repetir, em que se avaliava a exatidão dos parâmetros querológicos. Os valores de consistência interna variaram entre .60 e .96 , com valores médios para cada teste superiores a .80 (qr) e .70 (qe), 2); estabilidade temporal, pela técnica teste-reteste (intervalo de duas a três semanas) cujas correlaçóes (moderadas) de Spearman variaram entre .53 (qr) e .56 (qe); e 3) fiabilidade interrespondente nos testes expressivos, cujo valor de Spearman apontou uma correlação forte $(\mathrm{r}=.87)$ (Hermans et al., 2010). A validade de constructo foi avaliada pela correlação entre desempenho e características dos participantes: as correlaçóes (rho de Spearman) entre a idade das crianças e os resultados dos testes variaram entre .61 e .70; a correlação com o género revelou que o feminino superou o masculino. No que respeita à correlação relativa aos pais/cuidadores ouvintes em relação aos surdos, o estudo demonstrou que os filhos de pais surdos evidenciam mais facilidade na execução do teste. Os valores de Spearman apontarem relaçóes moderadas (.60 e .61, p<.001), na querologia expressiva e recetiva, respetivamente. Os autores constataram melhores desempenhos pelos participantes do género feminino ( $r=.21$ e $r=.34$ ), e o grupo de filhos de pais surdos $\left(r_{q r}=.41\right.$ e $r_{q e}=34$ ), tal como expectável (Herman \& Roy, 2006; Hermans et al., 2010; Maller et al., 1999). A validade preditiva foi analisada por Ormel (2008), na relação entre proficiência da língua gestual e competências de linguagem falada e escrita, que apontou para correlaçóes positivas e significativas (Mann, 2007).

Costa (2012), no Brasil, propôs a Avaliação Fonológica da Lingua Brasileira de Sinais: FONO-LIBRAS, avaliando quatro crianças surdas, entre os 6 e os 12 anos. O teste era constituído por 50 figuras distribuídas em várias categorias. As figuras eram mostradas no computador e as crianças, individualmente, eram estimuladas a enunciar o gesto de cada imagem. $\mathrm{O}$ teste procurava identificar os processos querológicos de assimilação, omissão, epêntese e metátese. As propriedades psicométricas do instrumento não são apontadas.

Os 19 itens do Phonological Awareness for American Sign Language objetivam perceber se os 87 adultos, entre 20 e 50 anos, surdos - divididos em três grupos consoante a sua exposição à língua gestual, são sensíveis aos parâmetros estruturais dos gestos selecionando o gesto correto com base em propriedades isoladas de movimento, localização ou configuração de imagens apresentadas (Corina $\&$ Welch, 2014). A análise da precisão do teste foi efetuada 
com base nos acertos vs. tentativas, bem como os testes estatísticos ANOVA para avaliar a variação do desempenho face ao nível de experiência ao idioma (nativo, precoce e tardio), o que se confirmou $(M=16,1$, IC 95\% [14,98, 17,22]).

Cruz (2016) com o Teste de Consciência Fonológica na Libras (TCF-Libras) avaliou o nível de consciência fonológica na Língua de Sinais de 34 crianças e adolescentes surdos bilíngues, entre os 9 e os 14 anos (com início da aquisição da Libras precoce se 1-4 anos, ou tardio depois dos 4 anos), e sete adultos com aquisição precoce, estudando a hipótese da influência de uma aquisição precoce ou tardia da língua de Sinais. O teste avalia os parâmetros da configuração da mão, localização e movimento em 45 itens, sendo nove itens exemplos e 36 itens-teste. Há três itens de prática em cada etapa do TCF-Libras, 15 itens-teste na avaliação do parâmetro da configuração da mão, 12 na avaliação do parâmetro da localização e nove na avaliação do parâmetro do movimento. Para cada item e parâmetro é apresentado um slide com quatro imagens: o "alvo", i.e., a imagem de referência, e outras três possibilidades de respostas, e o participante deve selecionar a que tem a mesma característica querológica da imagem-alvo. As propriedades psicométricas não são apontadas e os resultados são descritos em função dos acertos.

Em Portugal, Rodrigues (2017) desenvolveu o Teste de Avaliação da Compreensão da LGP para crianças surdas entre os 5 e 6 anos. O primeiro item, no âmbito da avaliação querológica - pseudogestos, constituído por 10 questóes, avalia a capacidade de discriminar a formação de gestos da LGP e de gestos que não pertencem ao léxico da LGP (pseudogestos). O segundo item (10 questóes) avalia a discriminação querológica das unidades mínimas, avaliando a capacidade de distinguir gestos semelhantes, com base no conhecimento do significante. Assim, são apresentados dois vídeos (pares mínimos) e uma imagem, devendo ser selecionado o vídeo que corresponde à imagem apresentada. As propriedades psicométricas não são apontadas, e a autora faz algumas apreciações (e.g.: dificuldade na perceção do próprio teste e nos domínios dos queremas da LGP) para posteriores reformulaçóes e validação. A literatura aponta a relação entre a experiência linguística inicial/momento/idade do início da exposição ao idioma (Freitas, 2004), bem como a qualidade e a quantidade do estímulo linguístico (Quadros \& Cruz, 2011).

Dada a escassez de evidências, e na inexistência de um instrumento de avaliação validado da consciência querológica no âmbito nacional, este artigo tem como objetivo a análise das propriedades psicométricas do instrumento criado para o efeito - Instrumento de Avaliação da Consciência Querológica da Língua Gestual Portuguesa (IACQ-LGP). Considera-se que a criação/utilização do IACQ-LGP, instrumento pioneiro na avaliação querológica da LGP, irá contribuir não só no desempenho escolar de todos os alunos surdos, uma vez que, antes de avaliar (a consciência querológica), será necessário haver um trabalho mais efetivo dela, mas também irá coadjuvar na intervenção de outros profissionais. Até então, e por não haver uma ferramenta métrica, a intervenção da LGP é realizada apenas a cargo da observação e das conclusôes dos professores. Por meio da aplicação do instrumento, os educadores poderão compreender (se e) quais as características linguísticas querológicas que estão desajustadas e intervir de uma forma mais concreta e eficaz. Esse objetivo fundamenta-se na necessidade da aplicação de instrumentos válidos e elaborados para o efeito (Landa \& Clark, 2019). 


\section{Método}

A amostra foi constituída por 23 alunos surdos que se comunicam por meio da LGP, entre os 5 e os 12 anos (9.09 \pm 1.92$)$, 17 do género masculino $(73,9 \%)$ e 6 do género feminino (26,1\%), havendo uma maior incidência de participantes com 9 e 10 anos $(n=14,60.8 \%)$. Os participantes frequentavam quatro escolas de referência para a educação bilíngue de alunos surdos, em diferentes zonas do país, e a maioria frequentava o $3^{\circ}$ ano de escolaridade $(\mathrm{n}=8$; $34.8 \%$ ), o que parece estar de acordo com a idade cronológica. A maioria das crianças participantes nasceram surdas $(\mathrm{n}=16 ; 69.6 \%)$ - com os restantes a serem diagnosticados até os 4 anos, tendo tido um primeiro contacto com a LGP a partir dos 3 anos ( $n=9 ; 39.1 \%$ ), idade com que iniciaram o ensino pré-escolar, apesar do contacto formal com a língua natural ( $\mathrm{n}=20 ; 87 \%)$. A maioria dos encarregados de educação dos participantes, por seu turno, não tem domínio da LGP ( $\mathrm{n}=20 ; 87 \%)$, apesar de $22(95.7 \%)$ aceitarem a LGP, e os participantes contactam pouco com pessoas que dominem a LGP $(\mathrm{n}=18 ; 78.3 \%)$.

O IACQ-LGP é um instrumento elaborado para avaliar a consciência querológica, de crianças surdas, entre os 5 e os 12 anos que se comuniquem por meio da LGP, e o seu desenvolvimento considerou a regra da boa-formação dos gestos (Cruz, 2018). A sua administração pode ser de caráter individual ou grupal. O IACQ-LGP está dividido em cinco subtestes: 1 - Avaliação da execução de 10 gestos (Figura 1): a avaliação das unidades mínimas é de caráter somático, quantificando o conhecimento dos avaliados na execução dos gestos solicitados. Esse subteste é constituído por 10 itens, em que o avaliado observa uma imagem de cada vez e procede à execução do seu gesto; o registo da execução, em cada uma das unidades mínimas, é realizado pelo avaliador na folha de registos, com a pontuação de 1 (realização correta de cada unidade mínima) ou 0 (não realiza/realiza incorretamente) pontos; 2 - Avaliação da unidade minima local de articulação: quantifica o conhecimento dos avaliados relativamente ao parâmetro local de articulação, tendo de selecionar a opção correta entre as várias apresentadas; 3 - Avaliação da unidade mínima do movimento: quantifica o conhecimento dos avaliados relativamente ao movimento, tendo de selecionar a opção correta entre as várias apresentadas; 4 - Avaliação da unidade minima da configuração da mão: implica o conhecimento sobre configuração da mão e a seleção da opção correta entre várias apresentadas. Esses subtestes (2, 3 e 4) são constituídos por cinco itens, mas, antes de dar início à avaliação propriamente dita, serão apresentados dois itens exemplos (que náo contabilizam no processo de avaliação), de forma a explicar ao avaliado o que é requerido. Os subtestes (Figura 2) são apresentados da seguinte forma: um gesto realizado com determinado local de articulação, movimento ou configuração da mão, e três opçóes de escolha (A, B e C) com gestos e o avaliado deverá escolher o/a gesto/ opção que apresenta o mesmo local de articulação do gesto apresentado; e 5-Discriminação de pares mínimos (Figura 3): na sequência dos anteriores, esse subteste é constituído por cinco itens com dois itens exemplos, sendo apresentada uma imagem e dois gestos (A e B) que apresentam um par mínimo, devendo o avaliado escolher o gesto correspondente. 


\section{Figura 1}

Exemplo do Teste da avaliação das unidades minimas: configuração da mão, movimento e local de articulação

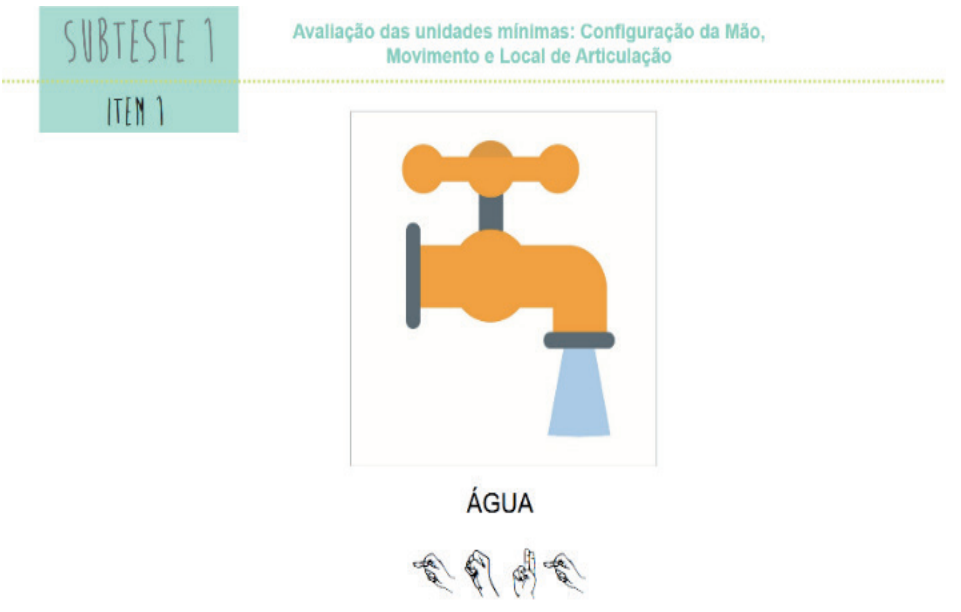

\section{Figura 2}

Exemplo do Teste da avaliação do local de articulação

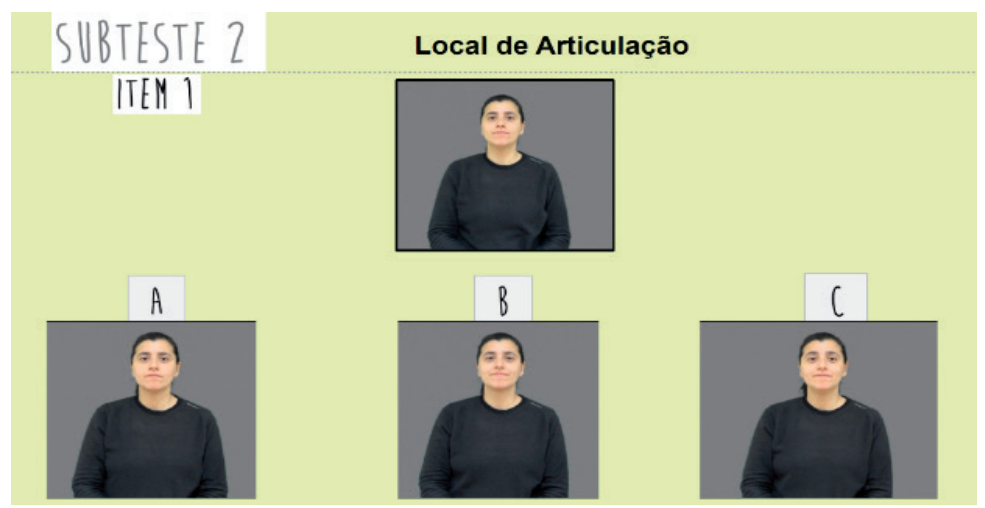

\section{Figura 3}

Exemplo do Teste da discriminação de pares mínimos

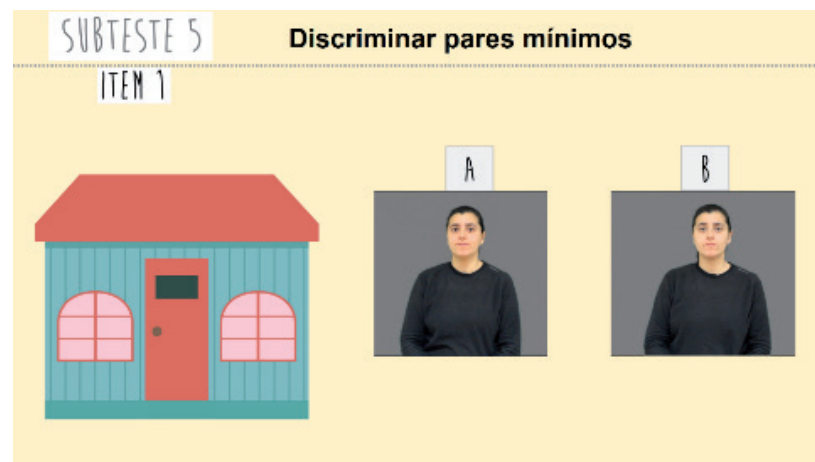


De acordo com as diretrizes para a construção ou adaptação de testes (Haug et al., 2016), foi criado um manual de apoio à aplicação do instrumento de avaliação onde estão descritas todas as informaçóes inerentes à sua utilização. No manual existem soluçóes referentes a cada subteste, devendo, o avaliador, conforme a prestação de cada avaliado, preencher as tabelas de registo para cada teste.

Todos os procedimentos éticos inerentes a uma investigação dessa natureza foram assegurados. Por meio de um consentimento informado, foram estabelecidos contactos com as direções das diferentes instituições onde se pretendia aplicar o IACQ-LGP, professores, encarregados de educação e respetivos educandos, requerendo a autorização e a participação no desenvolvimento do estudo. Foi ainda clarificado que a qualquer momento da investigação os participantes poderiam desistir, garantindo-se sempre a confidencialidade dos dados, bem como o anonimato deles.

A aplicação do teste foi realizada no estabelecimento escolar de cada participante, tendo cada aplicação uma duração média de 25 minutos, e recorreu-se à aplicação individual e em grupo. De uma forma geral, os avaliados demonstraram interesse na execução do teste e não revelaram cansaço durante a sua aplicação, apesar de alguma dificuldade na perceção dos subtestes 2, 3 e 4 . A versão 25 do software IBM Statistical Package of Social Sciences (SPSS) foi utilizada para o tratamento dos dados obtidos.

A construção do IACQ-LGP baseou-se nas linhas de orientação para o desenvolvimento, avaliação e utilização de testes de língua gestual, nomeadamente ao nível da consideração das evidências mais atuais bem como das características da LGP e da relevância da consciência querológica para o processo de desenvolvimento e de aprendizagem das crianças surdas (Haug et al., 2016). Ainda de acordo com as diretrizes, houve um cuidado especial em envolver, ao longo de todas as etapas, pessoas surdas com e sem formação académica, com o intuito de assegurar que os itens refletissem a estrutura da LGP, ao mesmo tempo que a seleção das imagens, visualmente acessíveis a crianças, também foi feita com base em critérios pré-estabelecidos e acordados entre peritos, bem como a seleção de itens representativos da consciência querológica (Haug et al., 2016). Importa referir que os gestos escolhidos fazem parte do léxico da LGP e têm variação dialetal residual; desse modo, os gestos são estáveis e a sua execução não é variável nem se prende com especificadores semânticos, de tamanho, forma ou outros.

As tarefas mais relevantes para a avaliação da consciência querológica passam pela identificação das configurações da mão, dos locais de articulação e dos movimentos com caráter distintivo (Karnopp, 1999), podendo ser avaliadas por meio da comparação entre dois pares mínimos ou da identificação de gestos com unidades mínimas iguais. Segundo a ordem de aquisição querológica, é consensual que o local de articulação seja o primeiro a ser adquirido, seguindo-se o movimento e a configuração de mão (Siedlecki \& Bonvillian, 1993). Desse modo, os subtestes 2, 3 e 4 foram colocados por essa ordem. Na construção do subteste 2, seguiu-se a ordem nivelada apresentada por Karnopp (1997): 10) espaço neutro, tronco, queixo e testa; $2^{\circ}$ ) mão e bochecha; $3^{\circ}$ ) meio da cara, pulso, pescoço, cabeça; $4^{\circ}$ ) antebraço; $5^{\circ}$ ) parte superior do braço. Da mesma forma, relativamente ao movimento e à configuração da mão, Carmo (2010) apresentou uma ordem de sua aquisição, e que também foram seguidos. Foi nessa base que o IACQ-LGP foi criado, surgindo no seguimento da carência já evidenciada e 
resultado da adaptação de vários testes já realizados (Costa, 2012; Cruz \& Lamprecht, 2008; Rodrigues, 2017). Os critérios de seleção do vocabulário consideraram os campos semânticos apreendidos mais facilmente pelas crianças (Carmo et al., 2007), sendo estes representados por gestos simples e compostos. As imagens contemplaram itens lexicais pertinentes ao léxico infantil, tendo sido priorizadas imagens coloridas e sem muitos estímulos (Costa, 2012).

Após a obtenção da versão final, foi fornecida uma cópia dela, bem como um questionário elaborado para o efeito, a sete peritos (Polit \& Beck, 2006) selecionados com base na sua experiência académica e profissional com a população em questão e no âmbito da validação de instrumentos: um dos elementos é perito na validação de instrumentos de avaliação, um intérprete de LGP, uma psicomotricista surda, uma terapeuta da fala com especialização em educação de surdos e três docentes de LGP, desenvolvendo a sua atividade profissional há pelo menos 5 anos. O questionário, elaborado para a avaliação do conteúdo, implicou que cada perito cotasse cada item quanto ao nível da sua relevância, clareza, simplicidade e ambiguidade. As opçóes de resposta variam entre 1=muito "irrelevante" até 4=muito "relevante", tendo sido explicado a todos os peritos qual o objetivo do estudo, os objetivos da escala e a população-alvo.

\section{Resultados}

A análise das propriedades métricas fornece evidências para a identificação das áreas fortes e fracas dos instrumentos de avaliação (Rust \& Golombok, 2009), pelo que os estudos dessa natureza devem assentar os seus princípios na validade, i.e., se um instrumento mede o que se pretende medir e na fiabilidade ou grau que define a ausência de erro aleatório dos instrumentos (Haug, 2005; Haug et al., 2016). No âmbito da validade, há de analisar-se a validade de conteúdo, critério e constructo (Haug, 2005).

A validade de conteúdo implica a avaliação sistemática dos conteúdos dos itens e da escala para ajuizar a representatividade do constructo (Yahgmaie, 2003). Em uma primeira fase, com base na revisão extensiva da literatura e, em seguida, em uma abordagem mais empírica, em que foi elaborado um questionário para que sete peritos classificassem os itens. Para o efeito, foram calculados os índices de validade de conteúdo (IVC - Tabela 1), a proporção de acordo entre peritos (Tabela 2) e o Kappa de Kohen (Tabela 3).

\section{Tabela 1}

Indice de validade de conteúdo dos itens (IVC-I) do IACQ-LGP

\begin{tabular}{|c|l|c|c|c|c|}
\hline \multicolumn{2}{|c|}{} & Relevância & Clareza & Simplicidade & Ambiguidade \\
\hline Substeste 1 & Item 1 a Item 10 & 1 & 1 & 1 & 1 \\
\hline \multirow{5}{*}{ Subteste 2 } & Exemplo 1 & .86 & .86 & .86 & .86 \\
& Exemplo 2 & 1 & 1 & 1 & 1 \\
\cline { 2 - 6 } & Item 1 & 1 & 1 & .86 & .71 \\
\cline { 2 - 6 } & Item 2 & 1 & 1 & 1 & .86 \\
\cline { 2 - 6 } & Item 3 & 1 & .86 & .71 & .86 \\
\cline { 2 - 6 } & Item 4 e 5 & 1 & 1 & 1 & 1 \\
\hline
\end{tabular}




\begin{tabular}{|c|c|c|c|c|c|}
\hline \multirow{6}{*}{ Subteste 3} & $\begin{array}{l}\text { Exemplo } 1 \\
\text { Exemplo } 2\end{array}$ & $\begin{array}{l}1 \\
1\end{array}$ & $\begin{array}{c}1 \\
.86\end{array}$ & $\begin{array}{c}1 \\
.86 \\
\end{array}$ & $\begin{array}{c}1 \\
.86\end{array}$ \\
\hline & Item 1 & 1 & .86 & .86 & 1 \\
\hline & Item 2 & .86 & .71 & .71 & .71 \\
\hline & Item 3 & 1 & .86 & .71 & .71 \\
\hline & Item 4 & 1 & 1 & .86 & .86 \\
\hline & Item 5 & 1 & 1 & 1 & 1 \\
\hline \multirow[t]{2}{*}{ Subteste 4} & $\begin{array}{l}\text { Exemplo } 1 \text { e } 2 \\
\text { Item } 1\end{array}$ & $\begin{array}{c}1 \\
.86 \\
\end{array}$ & $\begin{array}{c}1 \\
.86 \\
\end{array}$ & $\begin{array}{c}1 \\
.86 \\
\end{array}$ & $\begin{array}{c}1 \\
.86 \\
\end{array}$ \\
\hline & Item $2,3,4$ e 5 & 1 & 1 & 1 & 1 \\
\hline \multirow{4}{*}{ Subteste 5} & $\begin{array}{l}\text { Exemplo } 1 \text { e } 2 \text {; } \\
\text { Item } 1 \text { e } 2\end{array}$ & 1 & 1 & 1 & 1 \\
\hline & Item 3 & 1 & 1 & 1 & .71 \\
\hline & Item 4 & 1 & .86 & 1 & .86 \\
\hline & Item 5 & 1 & 1 & 1 & 1 \\
\hline \multicolumn{2}{|c|}{ AU/IVC-E } & .99 & .96 & .95 & .94 \\
\hline \multicolumn{2}{|c|}{ M/IVC-E } & .99 & .96 & .95 & .94 \\
\hline
\end{tabular}

\section{Tabela 2}

Proporção de acordo entre peritos

\begin{tabular}{lcccc}
\hline & Relevância & Clareza & Simplicidade & Ambiguidade \\
\hline P1 & 1 & 1 & .97 & .97 \\
\hline P2 & 1 & 1 & 1 & 1 \\
\hline P3 & 1 & .97 & 1 & 1 \\
\hline P4 & 1 & .95 & .89 & .89 \\
\hline P5 & 1 & 1 & 1 & .97 \\
\hline P6 & 1 & .97 & .97 & .95 \\
\hline P7 & .92 & .84 & .84 & .82 \\
\hline
\end{tabular}

Tabela 3

Valores de Kappa de Cohen

\begin{tabular}{|c|c|c|c|c|c|c|c|}
\hline Peritos & $\mathbf{1}$ & $\mathbf{2}$ & $\mathbf{3}$ & $\mathbf{4}$ & $\mathbf{5}$ & $\mathbf{6}$ & $\mathbf{7}$ \\
\hline 1 & 1 & & & & & & \\
\hline 2 & .99 & 1 & & & & & \\
\hline 3 & .98 & .99 & 1 & & & & \\
\hline 4 & .93 & .93 & .93 & 1 & & & \\
\hline 5 & .98 & .99 & .99 & .93 & 1 & & \\
\hline 6 & .96 & .97 & .93 & .93 & .97 & 1 & \\
\hline 7 & .87 & .87 & .86 & .84 & .86 & .85 & 1 \\
\hline
\end{tabular}


Todos os itens foram considerados representativos, com índices de validade de conteúdo (IVC) superiores a .80, exceção aos cinco itens assinalados na tabela, mas que, como superiores a .70, foram mantidos, considerando-se algumas sugestóes dos peritos, com reformulaçóes para uma maior clareza das instruçóes e das imagens. Situação idêntica ocorre com o acordo universal e médio, com valores superiores a .90 (Polit \& Beck, 2006). As proporçóes de acordo entre os peritos revelam, no geral, elevada concordância, assinalando-se apenas valores de .82 e 84 nos critérios de ambiguidade e simplicidade, envolvendo o mesmo perito (7). Os valores de Kappa variam entre 1 e -1 , com valores positivos a indicar o acordo entre peritos, e variando de acordo com: acordos fracos se Kappa inferior a .40, e moderados se .40<K<.60, bons se entre $.40<\mathrm{K}<.60$ e excelente se superior a .75 (Wynd et al., 2003), apesar de Viera e Garret (2005) apontarem um acordo substancial com valores entre .61 e .80 ou perfeito se $\mathrm{k}>$.81. Os valores do Kappa de Cohen reportam a tendência para acordos perfeitos (k>.84).

A análise da fiabilidade focou-se em dois índices (Tabela 4): estabilidade temporal com a aplicação do teste ao mesmo indivíduo $(\mathrm{n}=15)$ com 2 a 3 semanas de intervalo, pela técnica do teste-reste, por meio do cálculo dos coeficientes de correção de Spearman, cujos valores são considerados como excelentes se superiores a .90 , elevados se entre .70 e .89 e moderados se entre .40 e .69 (Pestana \& Gageiro, 2008); e a consistência interna (alpha de Cronbach) do teste com a amostra total com valores excelentes se $\alpha>.80$, adequados se entre .70 e .80 e inadequados se inferiores a .70 (Noonan et al., 2009). Os valores são apresentados por item, exceção apenas quando os subitens reportam valores diferentes. Observam-se valores elevados do coeficiente alpha $(\alpha>.78)$, indicando a sua consistência interna, mesmo com uma amostra reduzida e os coeficientes de Spearman parecem apontar para correlaçóes moderadas a fortes, exceção feita no subteste 5 cujo valor $(r=.13)$ indicia uma correlação fraca.

\section{Tabela 4}

A fiabilidade dos itens IACQ-LGP

\begin{tabular}{|c|c|c|c|}
\hline \multicolumn{2}{|c|}{ Subtestes e itens IACQ-LGP } & $\begin{array}{c}\mathrm{r}(\mathrm{n}=15) \\
\text { (teste-reteste) }\end{array}$ & $\alpha$ (consistência interna); $\mathrm{n}=23$ \\
\hline \multirow{10}{*}{ Subteste 1} & Item 1 & 1 & .80 \\
\hline & Item 2 & .68 & .80 \\
\hline & Item 3 & .63 & .80 \\
\hline & Item 4 & .68 & .80 \\
\hline & Item 5 & .54 & .80 \\
\hline & Item 6 & .42 & .80 \\
\hline & Item 7 & .70 & .80 \\
\hline & Item 8 & .37 & .79 \\
\hline & Item 9 & .68 & .80 \\
\hline & Item 10 & .49 & .79 \\
\hline \multicolumn{2}{|c|}{ Subteste 2} & .32 & .80 \\
\hline \multicolumn{2}{|c|}{ Subteste 3} & .37 & .80 \\
\hline \multicolumn{2}{|c|}{ Subteste 4} & .73 & .80 \\
\hline \multicolumn{2}{|c|}{ Subteste 5} & .13 & .80 \\
\hline
\end{tabular}


A validade de critério não foi analisada devido à inexistência de outras medidas; e a validade de constructo, i.e.: a relação entre o instrumento e o conceito teórico que se analisa, foi analisada por meio das intercorrelaçóes entre os vários itens/domínios constituintes do teste (Tabela 5), que se devem caracterizar pela sua autossuficiência. Tal como se constata, os valores de Spearman apontam para correlaçóes fracas a moderadas.

\section{Tabela 5}

Correlação entre os subtestes do IAQ-LGP

\begin{tabular}{|c|c|c|c|c|c|c|}
\hline Subtestes & S1 & S2 & S3 & S4 & S5 & Total \\
\hline S1 & 1 & $.44^{*}$ & .22 & $.54^{* *}$ & .12 & $.84^{* *}$ \\
\hline S2 & & 1 & .22 & $.57^{* *}$ & $.51^{*}$ & $.69^{* *}$ \\
\hline S3 & & & 1 & .36 & .30 & $.47^{*}$ \\
\hline S4 & & & & 1 & .46 & $.79^{* *}$ \\
\hline S5 & & & & & 1 & $.50^{*}$ \\
\hline $\begin{array}{l}\text { Total } \\
{ }^{*} \mathrm{p}<.05 ; \\
{ }^{* *} \mathrm{p}<.01\end{array}$ & & & & & & 1 \\
\hline
\end{tabular}

A análise fatorial exploratória (Tabela 6) investigou a dependência de um conjunto de variáveis observadas, explicitando a interrelação entre elas, procurando identificar novos fatores e estabelecer dimensóes (Pestana \& Gageiro, 2008). Para esse efeito, foram assumidos os seguintes procedimentos: rotação varimax, eigenvalues superior a 1.0 e um mínimo de $10 \%$ de variância explicada por cada componente, com pesos fatoriais de pelo menos .40. Os primeiros três fatores explicam $40.9 \%$ da variância total e parece inferir-se a estrutura multidimensional do instrumento, que carece, contudo, de uma análise mais aprofundada (e.g.: análise fatorial confirmatória) com uma amostra significativa e representativa. Um dos requisitos para o desenvolvimento de teste que avaliem a proficiência linguística implica a existência de relaçóes significativas entre os resultados globais e a idade da criança, pelo que se analisaram as correlaçóes de Spearman, com valores tendencialmente moderados $(>.30, \mathrm{p}<.01)$, bem como com o ano de escolaridade $(\mathrm{r}=.47, \mathrm{p}<.01)$. O género náo apresenta diferenças no desempenho dos itens com correlaçóes fracas a moderadas nos subtestes $(.02>\mathrm{r}<.37)$. Situação idêntica constata-se na análise da correlação da idade de aquisição da LGP $(.08>\mathrm{r}<.30)$ e do domínio desta língua pelos cuidadores $(.05>\mathrm{r}<.55)$, com diferenças significativas apenas nos itens cavalo e carro no subteste 1 . 


\section{Tabela 6}

\section{Análise fatorial exploratória}

\begin{tabular}{|c|c|c|c|c|c|c|c|c|c|}
\hline Subtestes IAQ-LGP & F1 & F2 & F3 & F4 & F5 & F6 & F7 & F 8 & F 9 \\
\hline S1_água_configuração & & & .78 & & & & & & \\
\hline S1_água_movimento & & & .78 & & & & & & \\
\hline S1_água_local de articulação & & & .78 & & & & & & \\
\hline S1_cavalo_configuraçáo & .59 & & & & & & & & \\
\hline S1_cavalo_movimento & .59 & & & & & & & & \\
\hline S1_cavalo_local articulação & .59 & & & & & & & & \\
\hline S1_leite_configuração & & .84 & & & & & & & \\
\hline S1_leite_movimento & & .84 & & & & & & & \\
\hline S1_leite_local de articulação & .50 & & & & & & & & \\
\hline S1_amarelo_configuração & & & & & & & & & .65 \\
\hline S1_amarelo_movimento & & .78 & & & & & & & \\
\hline S1_amarelo_local articulação & & & -.56 & & & & & & \\
\hline S1_casa_configuração & & .78 & & & & & & & \\
\hline S1_casa_movimento & & .78 & & & & & & & \\
\hline S1_casa_local articulação & & .78 & & & & & & & \\
\hline S1_carro_configuração & & & & & & & -.75 & & \\
\hline S1_carro_movimento & & & & & & & & .46 & \\
\hline S1_carro_local articulação & & & & -.56 & & & & & \\
\hline S1_ananás_configuração & .72 & & & & & & & & \\
\hline S1_ananás_movimento & .69 & & & & & & & & \\
\hline S1_ananás_local articulação & .72 & & & & & & & & \\
\hline S1_faca_configuração & .65 & & & & & & & & \\
\hline S1_faca_movimento & .70 & & & & & & & & \\
\hline S1_faca_local articulação & .70 & & & & & & & & \\
\hline
\end{tabular}




\begin{tabular}{|c|c|c|c|c|c|c|c|c|c|}
\hline Subtestes IAQ-LGP & F1 & F2 & F3 & F4 & F5 & F6 & F7 & F 8 & F 9 \\
\hline S1_árvore_movimento & & & & .41 & & & & & \\
\hline S1_árvore_local articulação & & & -.56 & & & & & & \\
\hline S1_pássaro_configuração & & & .64 & & & & & & \\
\hline S1_pássaro_movimento & & & .64 & & & & & & \\
\hline S1_pássaro_local articulação & & & .64 & & & & & & \\
\hline S2_Local de articulação_1 & .39 & & & & & & & & \\
\hline S2_Local de articulação_2 & .49 & & & & & & & & \\
\hline S2_Local de articulaçáo_3 & & .50 & & & & & & & \\
\hline S2_Local de articulação_4 & & & .48 & & & & & & \\
\hline S2_Local de articulação_5 & & & & .71 & & & & & \\
\hline S3_Movimento_1 & .60 & & & & & & & & \\
\hline S3_Movimento_2 & & & .56 & & & & & & \\
\hline S3_Movimento_3 & .32 & & & & & & & & \\
\hline S3_Movimento_4 & & & & & & -.54 & & & \\
\hline S3_Movimento_5 & .55 & & & & & & & & \\
\hline S4_Configuração_1 & & & & .49 & & & & & \\
\hline S4_Configuração_2 & & & .50 & & & & & & \\
\hline S4_Configuração_3 & .69 & & & & & & & & \\
\hline S4_Configuração_4 & .80 & & & & & & & & \\
\hline S4_Configuração_5 & .62 & & & & & & & & \\
\hline S5_Pares mínimos_1 & & & & & .63 & & & & \\
\hline S5_Pares mínimos_2 & & .50 & & & & & & & \\
\hline S5_Pares mínimos_3 & & & & & .51 & & & & \\
\hline S5_Pares mínimos_4 & & .50 & & & & & & & \\
\hline S5_Pares mínimos_5 & .50 & & & & & & & & \\
\hline
\end{tabular}




\section{Discussốes}

Para além da nova concetualização sobre as pessoas surdas (Pratas et al., no prelo), a educação das crianças, em Portugal, bem como em outros países, tem conhecido um interesse crescente e com alteraçóes significativas, com a introdução da LGP em contexto escolar e em uma aprendizagem bilíngue (Carmo et al., 2007), em detrimento do processo educativo exclusivamente baseado na linguagem oral. A avaliação desses processos deve ser feita com instrumentos válidos e fiáveis (Landa \& Clark, 2019), fundamentando a pertinência deste estudo, dada a escassez de instrumentos validados e a tendência para a análise qualitativa apenas centrada no número de acertos (Costa, 2012; Cruz \& Lamprecht, 2008; Rodrigues, 2017).

Em uma primeira fase, procedeu-se à análise extensiva da literatura relativamente à LGP e à consciência querológica e aos instrumentos na área (Costa, 2012; Cruz \& Lamprecht, 2008; Karnopp, 1999; Siedlecki \& Bonvillian,1993; Rodrigues, 2017), para a criação do IACQ-LGP. Com base nesta revisão, foi elaborado o instrumento que foi submetido à avaliação de peritos na área, no sentido de aprovarem (ou não) a representatividade dos itens, bem como se a sua forma de apresentação era simples, clara e pouco ambígua. Os itens foram considerados como representativos, tendo sido classificados como relevantes/muito relevantes.

Apenas cinco itens (presentes no subtestes 2, 3 e 5) indicaram valores inferiores (.71) ao nível da simplicidade, clareza e ambiguidade, pelo que se procederam às respetivas reformulaçôes, decidindo-se pela sua manutenção. Situação idêntica ocorre ao nível do acordo universal e da média da escala, indiciando a validade de conteúdo do instrumento. Apesar da capacidade querológica ser um constructo recente, e dependente da maturação das várias etapas do desenvolvimento cognitivo e intelectual da criança, bem como das experiências linguísticas, e ainda da motricidade fina (Karnopp, 1999), os peritos consideraram a sua relevância para a avaliação da aprendizagem e domínio da LGP, sustentando a sua importância na relação entre a aprendizagem e aquisição desta língua com a aprendizagem da leitura em um código alfabético (Mann, 2007). Não se conhecem outros estudos que tenham procedido a esse tipo de análise. Há apenas a referir que o Perito 7 (técnico surdo, com conhecimento da língua, mas não propriamente do seu processo de ensino-aprendizagem) apontou valores inferiores ao nível da clareza, simplicidade e da ambiguidade de alguns itens, mas cujos valores foram adequados (>.82). A concordância entre peritos foi excelente, realçando-se a importância das diferenças regionais da LGP (Correia, 2009), incluindo um perito residente na região autónoma dos Açores.

Com base nos comentários dos peritos, a versão final do IACQ-LGP foi aplicada e para a análise da estabilidade temporal o instrumento foi aplicado a 15 participantes com 2-3 semanas de intervalo, obtendo-se correlaçóes moderadas a fortes, indiciando a relação, mas não sobreposição, entre os diferentes itens e subtestes (Hermans et al., 2010). Nesse âmbito, há de destacar-se o subteste 4, com valores mais elevados de correlação, eventualmente justificados pela maior clareza, interesse e facilidade de compreensão aquando dos itens-exemplo, explicando ainda a necessidade de uniformização do parâmetro da configuração da mão na aprendizagem da LGP (Carmo et al., 2007). Apenas o subteste 5 apresentou valores indicativos de correlaçóes fracas, pelo que deverá ser reequacionado. Ao analisarmos o número de acertos das duas aplicações, constata-se uma evolução do desempenho, o que pode ter concorrido para os valores inferiores. Assim, apesar da suposta sensibilidade desde cedo aos queremas (Basso, 
2006), infere-se que a exposição e experiência com a LGP (Corina \& Welch, 2014) poderá influenciar esse resultado.

O instrumento parece apresentar consistência interna, corroborando outros testes de língua gestual (Hermans et al., 2010), apesar da tendência para valores inferiores neste estudo (Hermans et al., 2010; Maller et al., 1999). Apesar da fiabilidade do IACQ-LGP ser aceitável, Nunnally (1976) aponta que, para efeitos de decisóes clínicas, esses valores devem ser superiores a .90. No entanto, esse valor é influenciado pela amostra, pelo que a aplicação do instrumento a uma amostra representativa e significativa pode influenciar os valores obtidos neste estudo. Os valores obtidos indiciam a fiabilidade do IACQ-LGP.

A validade de constructo foi analisada com base nas correlaçóes entre subtestes, bem como na análise fatorial exploratória, no sentido de se prever a estrutura fatorial do instrumento. Os resultados obtidos parecem apontar para a sua validade, prevalecendo as correlaçóes moderadas. No caso do subteste 1, destaca-se, como esperado, a correlação moderada com os subtestes 2 e 4. Por outro lado, a correlação fraca com os subtestes 3 e 5 poderá ser explicada pelo subteste 1 estar mais associado à querologia expressiva e os outros à recetiva (Hermans et al., 2010). Na solicitação do subteste 1 (querologia expressiva), não há uma exigência total de reflexão sobre as unidades mínimas semelhante à solicitada na recetiva (subtestes 2, 3, 4 e 5 - o avaliado tem de refletir na unidade mínima em questão), o que remete à perceção da forma como o desenvolvimento querológico se processa - progressão de grandes para as pequenas unidades (Carrol et al., 2003). O subteste 2 , pelo parâmetro que avalia, parece ser o primeiro a ser adquirido aquando da aquisição e desenvolvimento da consciência querológica (Siedlecki \& Bonvillian, 1993).

Tal como expectável, são notadas correlaçôes tendencialmente moderadas e significativas entre os vários itens, o que parece estar de acordo com a premissa que não avaliam as mesmas competências, mas que, de alguma forma, se relacionam para o desempenho querológico. Dessa forma, infere-se a relevância dos itens e subtestes do IACQ-LGP na avaliação da consciência querológica, em consequência das alterações que tendem a surgir nos queremas quando as crianças surdas expressam os primeiros gestos, demonstrando configuraçóes ou movimentos alterados (Carmo et al., 2007).

Finalmente, e no âmbito da AFE, procurou identificarem-se os fatores relevantes e com significado teórico (Matsunaga, 2010). Apesar da expectativa da estrutura ser bidimensional (expressiva e recetiva), a matriz estrutural obtida parece apontar para uma organização multidimensional, relevando-se os primeiros quatro fatores que explicam $40.8 \%$ da variância total, o que poderá querer dizer que as dimensóes poderão antes ser: configuração da mão, local de articulação, movimento e direção, inferindo-se que a direção que o gesto assume acarreta, também, um aspeto morfológico (Carmo, 2010). Tal como se constata, existem cinco fatores constituídos apenas por um ou dois itens, que, apesar de não terem sido eliminados nessa primeira abordagem, exigem uma atenção especial em uma investigação futura. Os valores obtidos nesta análise parecem evidenciar a validade de constructo.

A correlação moderada com a idade (Hermans et al., 2010; Maller et al., 1999) e com o (3) ano de escolaridade, aponta para a sua eventual influência na consciência querológica (Quadros \& Cruz, 2011), corroborando a importância do tempo de exposição à língua natural das crianças surdas e seu desenvolvimento consistente com especial atenção aos parâmetros 
gestuais (Stokoe, 1960) e não apenas ao seu significado global de um gesto (Karnopp, 1997). No nosso estudo, não existem diferenças significativas entre os grupos mais velhos, constatando-se apenas com as mais novas (Herman et al., 1999). O género não parece influenciar a consciência querológica, e os filhos de pais surdos apenas parecem ter um melhor desempenho em dois itens do subteste 1, parecendo não corroborar a ideia da melhor proficiência em todos os níveis (Herman \& Roy, 2006; Hermans et al., 2010; Maller et al., 1999). O período temporal da aquisição (precoce ou tardia) não parece revelar diferenças, apesar de eventualmente influenciado pela desigualdade dos participantes.

Por fim, e já antes evidenciado por Rodrigues (2017), será importante relacionar a dificuldade dos avaliados na perceção da execução dos subtestes 2, 3 e 4, corroborando o panorama nacional, em que, pelo facto de os pais dos alunos avaliados serem ouvintes, poderá atrasar o contacto com a língua natural, que acontece apenas em contexto escolar formal.

\section{Conclusóes}

A utilização de um instrumento de avaliação não pode ser baseada meramente ou na tradução de instrumentos existentes em outros países e culturas, pois a sua elaboração exige a análise da fiabilidade e validade dos domínios e itens, visando a sua operacionalização com a população a que se destina. Nesse sentido, e tal como Wassel (2016) afirma, há necessidade de um conjunto de procedimentos rigorosos, razão pela qual se dedicou particular atenção à validade de conteúdo, no sentido de se selecionarem os itens mais representativos do constructo em análise - a consciência querológica.

Os resultados obtidos forneceram evidências para a continuação do estudo, cujos resultados também positivos, permitiram inferir que o IACQ-LGP parece ser um instrumento a considerar na avaliação da consciência querológica de crianças surdas entre os 5 e os 12 anos, fornecendo informação e pistas para a intervenção na área com essas crianças, e com eventuais contributos robustos no seu processo educativo, sobretudo na aquisição da LGP e da língua portuguesa escrita, enquanto segunda língua. A aplicação do IACQ-LGP deveria ser feita em complementaridade com a recolha de outras informaçóes pertinentes, no sentido de uma recolha de dados mais abrangente ao nível da LGP, porém observa-se a inexistência de testes validados. Os docentes de LGP deverão explorar a consciência querológica como estratégia na promoção da alfabetização de seus alunos (Crume, 2013).

No confronto com os critérios de Haug (2005), há a destacar, também, os que não foram conseguidos: a não inclusão de diferentes grupos etários (e.g.: adolescentes e adultos, surdos e ouvintes) e o estabelecimento de normas no âmbito nacional, pelo que essas questóes deverão ser endereçadas em investigaçóes futuras. Acresce, ainda, a necessidade de análise de outros tipos de validade (e.g.: concorrente e preditiva), apesar de se considerar que o objetivo deste artigo foi alcançado, com impacto na investigação e prática no campo das pessoas surdas e do desenvolvimento da LGP. A relação entre proficiência na LGP e língua falada e escrita também deverá ser explorada. A forma de ensino da LGP deve ser repensada, pois é abordada apenas formalmente, em tempos escolares, sendo evidenciada a dificuldade na perceção dos participantes aos subtestes 2, 3 e 4. Por meio da avaliação dessa consciência, os professores de LGP conseguirão conduzir 
para um melhor processo de ensino-aprendizagem da língua natural das crianças surdas de forma a colmatar algumas dificuldades potenciadas pelo atraso do ensino da língua.

Este estudo preliminar, tal como qualquer outra investigação dessa natureza, apresenta algumas limitaçóes como a amostra reduzida e o facto de circunscrever-se a um intervalo de idade, havendo necessidade de aplicação futura do teste a uma amostra significativa e representativa do grupo de crianças surdas (e ouvintes) portuguesas, para a validação do instrumento por meio de técnicas estatísticas mais robustas (e.g.: análise fatorial confirmatória) que permitam a obtenção dos valores normativos consoante diferentes variáveis (e.g.: por género, escalão etário, etc.). A identificação e a análise das relaçóes entre a consciência querológica e variáveis pessoais (e.g.: idade, género, idade de aquisição da LGP) e contextuais (filhos de pais surdos e ouvintes, estatuto socioeconómico, relação com leitura e escrita, entre outros) permitirá um melhor conhecimento dos preditores a considerar e a tomada de decisóes (mais) precoces. A aplicação do instrumento em contexto escolar deverá ser outro passo a concretizar, bem como a eventual formação de profissionais para a sua administração e interpretação.

\section{REFERÊNCIAS}

Basso, F. (2006). A estimulação da consciência fonológica e a sua repercussão no processo de aprendizagem da lecto-escrita. Dissertação de Mestrado, Universidade Federal de Santa Maria, Santa Maria, Rio Grande do Sul, Brasil.

Carmo, H., Martins, M., Morgado, M., \& Estanqueiro, P. (2007). Programa curricular de lingua gestual portuguesa educação pré-escolar e ensino básico. Ministério da Educação e Direção Geral de Inovação e de Desenvolvimento Curricular.

Carmo, P. (2010). Aquisição da língua gestual portuguesa: estudo longitudinal de uma criança surda dos 10 aos 24 meses. Dissertação de Mestrado, Universidade Católica Portuguesa, Lisboa, Portugal.

Carrol, J., Snowling, M., Hulme, C., \& Stevenson, J. (2003). The development of phonological awareness in pre-school children. Developmental Psychology, 5(39), 913-923. https:// doi.org/10.1037/0012-1649.39.5.913

Corina, D., \& Welch, S. (2014). Phonological Awareness for American Sign Language. The Journal of Studies and Deaf Education, 19(4), 530-545. https://doi.org/10.1093/deafed/enu023

Correia, I. (2009). O parâmetro expressão na Língua Gestual Portuguesa: unidade suprassegmental. Exedra, Revista Cientifica da Escola Superior de Educação de Coimbra, 1, 57-68.

Costa, R. (2012). Proposta de Instrumento para a Avaliação Fonológica da Lingua Brasileira de Sinais: FONOLIBRAS. Dissertação de Mestrado, Universidade Federal da Bahia, Salvador, Bahia, Brasil.

Crume, K. (2013). Teachers perceptions of promoting sign language phonological awareness in an ASL/English bilingual program. Journal of deaf studies and deaf education, 18, 464-488. https:// doi: $10.1093 /$ deafed/ent023

Cruz, C., \& Lamprecht, R. (2008). Proposta de instrumento de avaliação da consciência fonológica, parâmetro configuração de mão, para crianças surdas utentes da Língua de Sinais Brasileira. Letras de hoje, 43(83), 98-106. 
Cruz, C. (2016). Consciência fonológica na lingua de sinais brasileira (libras) em crianças e adolescentes surdos com inicio da aquisiçâo da primeira lingua (libras) precoce ou tardio. Tese de Doutorado, Universidade Federal do Rio Grande do Sul, Porto Alegre, Rio Grande do Sul, Brasil.

Cruz, C. (2018). Consciência fonológica da língua de sinais: implicaçóes na linguagem e na leitura. Revel, 15, edição especial, 63-82.

Freitas, C. (2004). Sobre a consciência fonológica. In R. Lamprecht (Ed.), Aquisição Fonológica do Português: Perfil de Desenvolvimento e Subsídios para a Terapia (pp. 177-192). Artmed.

Haug, T. (2005). Review of sign language assessment instruments. Sign Language \& Linguistics, 8, 61-98.

Haug, T., Mann, W., Boers-Visker, E., Contreras, J., Enns, C., Herman, R., \& Rowley, K. (2016). Guidelines for sign language test development, evaluation, and use. Recuperado em 20 agosto de 2020 de http://www.signlang-assessment.info/

Herman, R., Holmes, S., \& Woll, B. (1999). Assessing BSL development-Receptive skills test. Forest Bookshop.

Herman, R., \& Roy, P. (2006). Evidence from the wider use of the BSL receptive skills test. Deafness and Education International, 8, 33-47. https://doi.org/10.1002/dei.33

Hermans, D., Knoors, H., \& Verhoeven, L. (2010). Assessment of Sign Language Development: The Case of Deaf Children in the Netherlands. Journal of Deaf Studies and Deaf Education, 15(2), $107-$ 119, 2010. https://doi.org/10.1093/deafed/enp030

Karnopp, L. (1997). Aquisição fonológica da língua brasileira de Sinais. Letras de Hoje, 32(4), 147-162.

Karnopp, L. (1999). Aquisição fonológica da língua brasileira de Sinais: estudo longitudinal de uma criança surda. Dissertação de Doutorado, Universidade Católica do Rio Grande do Sul, Porto Alegre, Rio Grande do Sul.

Landa, L., \& Clark, D. (2019). L2/Ln Sign Language Tests and Assessment Procedures and Evaluation. Psychology, 10, 181-198. https://doi.org/10.4236/psych.2019.102015

Lima, R. (2011). Fonologia Infantil: Aquisição, Avaliação e Intervençâo. Almedina.

Maller, S., Singleton, J., Suppalla, S., \& Wix, T. (1999). The development and psychometric properties of the American Sign Language Proficiency Assessment (ASL-PA). Journal of Deaf Studies and Deaf Education, 4, 249-269. http://doi.org/10.1093/deafed/4.4.249

Mann, W. (2007). German deaf children's understanding of referential distinction in written German and German Sign Language. Educational \& Child Psychology, 24, 59-76.

Matsunaga, M. (2010). How to factor-analyze your data right: Do's, don'ts, and how-to's. International Journal of Psychological Research, 3(1), 97-110.

Noonan, V., Miller, W., \& Noreau, L. (2009). A review of instruments assessing participation in persons with spinal cord injury. Spinal Cord, 47, 435-446. https://doi.org/10.1038/sc.2008.171

Nunnally, J. (1976). Psychometric Theory. New York: McGrawHill.

Ormel, E. (2008). Visual word recognition in bilingual deaf children. [Tese de Doutorado náo publicada]. University of Nijmegen, Nijmegen.

Pestana, M., \& Gageiro, J. (2008). Análise de dados para ciências sociais: A complementaridade do SPSS. 5a Edição. Ediçốes Sílabo. 
Polit, D., \& Beck, C. (2006). The content validity index: Are you sure you know what's being reported? Critique and recommendations. Research in Nursing \& Health, 29(5), 489-497. https://doi. org/10.1002/nur.20147

Pratas, M., Correia, I., \& Santos, S. (no prelo). Uma abordagem sobre a pessoa surda e a sua língua natural a Língua Gestual Portuguesa. Revista de Educação Especial e Reabilitação.

Quadros, R., \& Cruz, C. (2011). Lingua de sinais: instrumentos de avaliação. Artmed.

Rodrigues, R. (2017). Compreensão da Lingua Gestual Portuguesa em Crianças Surdas Proposta de um Instrumento de Avaliação. Dissertação de Mestrado, Escola Superior de Educaçáo Paula Frassinetti, Porto, Portugal.

Rust, J., \& Golombok, S. (2009). Modern Psychometrics. Routledge.

Siedlecki, T., \& Bonvillian, J. (1993). Location, handshape and movement: young children's acquisition of the formational aspects of American sign language. Sign language studies, 78, 31-52, 1993. https://doi/10.1353/sls.1993.0016

Stokoe, W. (1960). Sign and Culture: A reader for students of American Sign Language. Listok Press.

Viera, A., \& Garrett, J. (2005). Understanding interobserver agreement: The kappa statistic. Family Medicine, 37(5), 360-363.

Wassel, J. I. (2016). A review of Morais, Santos, and Lebre's (2016) "Psychometric properties of the Portuguese version of the Examen Geronto-Psychomoteur" and the critique of the influence of scholar literature: A call for greater responsibility. Educational Gerontology, 42(7), 513-515. https:// doi.org/10.1080/03601277.2016.1165067

Wynd, C., Schmidt, B., \& Schaefer, M. (2003). Two quantitative approaches for estimating content validity. Western Journal of Nursing Research, 25(5), 508-518. https://doi.org/10.1177/0193945903252998

Yaghmaie, F. (2003). Content validity and its estimation. Journal of Medical Education Spring, 3(1), 25-27.

Recebido em: 26/02/2020

Reformulado em: 10/08/2020

Aprovado em: 17/10/2020 
PRATAS, M.; CORREIA, I.S.C.; SANTOS, S. 